\title{
Agrupamento e Recomendação de Objetos de Aprendizagem no Padrão IEEE-LOM Considerando Estilos de Aprendizagem
}

\author{
Miller M. Mendes ${ }^{1}$, Vitor C. de Carvalho ${ }^{1}$, \\ Fabiano A. Dorça ${ }^{1}$, Rafael D. Araújo ${ }^{1}$, Renan G. Cattelan ${ }^{1}$ \\ ${ }^{1}$ Faculdade de Computação - Universidade Federal de Uberlândia (UFU) \\ Uberlândia - MG - Brasil.

\begin{abstract}
Repositories of educational materials grow larger as learning objects are created continuously, which makes the search for specific items a challenging task. In this way, such objects should be organized to better support a recommendation process. Thus, this paper presents an approach for clustering educational content from those repositories, based on learning styles. A comparative analysis of three different clustering algorithms was performed and promising results were obtained. Based on the results, a learning objects recommendation process is discussed.
\end{abstract}

Resumo. A grande quantidade de objetos de aprendizagem que são criados continuamente faz com que os repositórios de materiais educacionais fiquem cada vez maiores e a busca de itens específicos se torna um desafio. Dessa forma, faz-se necessário que tais objetos sejam organizados para que sua recomendação seja mais eficiente. Sendo assim, a proposta apresentada neste trabalho utiliza técnicas de clusterização para agrupar conteúdos educacionais em repositórios com base em estilos de aprendizagem. Uma análise comparativa de três diferentes algoritmos de agrupamento foi realizada e resultados promissores foram obtidos. Com base nos resultados, uma proposta de recomendação de objetos de aprendizagem é discutida.

\section{Introdução}

O avanço da computação em diferentes frentes tem criado sistemas cada vez mais dinâmicos e que se adaptam às necessidades dos usuários. Especialmente no contexto educacional, abordagens que visam melhorar a experiência de aprendizagem utilizando recursos de recuperação e personalização de conteúdo têm surgido. Em paralelo, o surgimento de Ambientes Virtuais de Aprendizagem (AVA) associado às premissas da Computação Ubíqua [Weiser 1999] tem potencializado a geração de Objetos de Aprendizagem (OAs) capturados e segmentados automaticamente [Araújo et al. 2016].

Os AVAs podem promover a interação entre sistemas, professores e estudantes ao utilizar linguagens e recursos tecnológicos para exibição de informação de forma organizada [Almeida 2003]. No meio acadêmico, o uso de AVAs tem ganho importância e, consequentemente, maior utilização [Torres and do Amaral 2011]. Dessa forma, adequar 
VI Congresso Brasileiro de Informática na Educação (CBIE 2017)

Anais do XXVIII Simpósio Brasileiro de Informática na Educação (SBIE 2017)

tais ambientes ao perfil do estudante, levando em conta suas características e individualidades, é uma necessidade real e, para tal, a utilização de OAs emerge como uma abordagem promissora [Audino and da Silva Nascimento 2012].

Repositórios já são utilizados para facilitar a busca de OAs a partir de suas diferentes áreas, conteúdos e tipos (áudio, vídeo, diagramas, texto, etc.). No entanto, observase que os repositórios de OAs possuem uma grande quantidade de dados, o que resulta em OAs que, muitas vezes, não atendem a um determinado perfil do aluno. A clusterização (ou agrupamento) é uma técnica que pode ser aplicada para aumentar a escalabilidade de sistemas de recomendação, na qual OAs podem ser agrupados utilizando métricas de similaridade. Assim, a recomendação de OAs pode ser aplicada em grupos específicos gerados em cada agrupamento.

Complementarmente, Estilos de Aprendizagem (EAs) são um conceito importante na criação de um sistema de recomendação e personalização de contéudo, justamente por sua relação direta com as possíveis estrategias de aprendizado adotadas pelos estudantes. Logo, recomendar conteúdo com base no perfil do estudante é uma abordagem promissora. As recomendações podem ser realizadas relacionando características individuais, contextuais e também com base nas ações do aluno quando este interage com o conteúdo [Zaina et al. 2012]. Cazella et al. [Cazella et al. 2009] propõem a criação de sistemas que possibilitam auxiliar o usuário na busca e seleção de um conteúdo com base em seu perfil. Um dos modelos mais aceitos é o de Felder e Silverman [Felder and Silverman 1988], que propõem um modelo de EAs com quatro dimensões: percepção, entrada, processamento e organização.

Assim, este trabalho propõe uma abordagem para o agrupamento e a recomendação de conteúdo educacional que leva em consideração a relação entre os EAs de Felder e Silverman e os metadados dos OAs (no formato IEEE-LOM). Utiliza-se uma estrategia para vetorizar os OAs, de modo a facilitar o processo de agrupamento e, com isso, possibilitar o emprego de técnicas de agrupamento para auxiliar o processo de recomendação. Entende-se que esta abordagem traga vantagens em termos de eficiência, uma vez que possibilita o pré-processamento das características dos EAs atendidos pelos OAs.

O restante do texto está organizado da seguinte forma: na Seção 2, são formalizados os fundamentos teóricos que embasam a proposta ora apresentada, que por sua vez, é detalhada na Seção 3; na Seção 4, é apresentada uma análise comparativa de três diferentes algoritmos de agrupamento, bem como os resultados obtidos; na Seção 5, são abordados alguns trabalhos relacionados, também de modo comparativo; e, finalmente, na Seção 6, são apresentadas as considerações finais.

\section{Fundamentação Teórica}

Esta seção engloba os principais conceitos associados à proposta ora apresentada.

\subsection{O Modelo de Estilos de Aprendizagem de Felder e Silverman (FSLSM)}

Estratégias de ensino que consideram as diferenças individuais dos estudantes têm sido estudadas há algum tempo, como é o caso de modelos de Estilos de Aprendizagem. [Riding and Rayner 2013] define Estilo de Aprendizagem (EA) como uma preferência in- 
VI Congresso Brasileiro de Informática na Educação (CBIE 2017)

Anais do XXVIII Simpósio Brasileiro de Informática na Educação (SBIE 2017)

dividual e habitual dos estudantes para com informações recebidas e processadas durante o processo de aprendizagem. Existem inúmeros modelos propostos na literatura.

O modelo proposto por Felder e Silverman [Felder and Silverman 1988], chamado de Felder-Silverman Learning Style Model (FSLSM) [Felder and Silverman 1988], foi utilizado nesta pesquisa, uma vez que é uma proposta amplamente utilizada em sistemas adaptativos para educação que representa os estilos de aprendizagem como uma tendência e não como características fixas. No modelo FSLSM, oito EAs são agrupados em quatro dimensões: percepção, que classifica os estudantes de acordo com a forma com que eles percebem o conteúdo (estilos Sensitivo e Intuitivo); entrada, indica a preferência para recepção do conteúdo (estilos Visual e Verbal); processamento, relacionada com a forma com que os estudantes participam e suas atitudes durante o processo de aprendizagem (estilos Ativo e Reflexivo); e, organização, que representa a preferência relacionada com a forma de organização do conteúdo (estilos Sequencial e Global).

\subsection{Relação entre Objetos de Aprendizagem e o modelo FSLSM}

Para que o conteúdo educacional possa ser personalizado individualmente, é necessário ter em mãos um conjunto de metadados que define cada material com informações relevantes. Nesse sentido, esse trabalho utiliza o padrão de metadados chamado de IEEELOM (Learning Object Metadada) proposto pelo comitê especial de padrões de tecnologias de aprendizagem (LTSC) da IEEE [Hodgins et al. 2002] para representar os metadados dos Objetos de Aprendizagem (OAs).

O IEEE-LOM possui mais de 50 campos de dados agrupados em nove categorias: Geral, Ciclo de Vida, Meta-Metadado, Técnico, Educacional, Direitos, Relação, Anotação e Classificação. A categoria Educacional é de fundamental importância para este trabalho, visto que apresenta as características educacionais e pedagógicas dos objetos de aprendizagem [Hodgins et al. 2002]. Com essas informações, trabalhos anteriores mapearam tais características com os estilos de aprendizagem do modelo FSLSM que mais se combinam [Dorça et al. 2016].

- Tipo de interatividade: O modo predominante de aprendizagem (puramente expositivo, exige participação ativa do estudante, ou misto) suportado pelo objeto de aprendizagem é indicado nesse campo. Assim, é possível verificar se um OA deve ser recomendado a um estudante Ativo ou Reflexivo.

- Nível de interatividade: Os valores armazenados neste campo indicam o quanto um OA é interativo, desde o nível muito baixo até o nível muito alto. Com isso, é possível identificar se um OA é mais apropriado para um estudante Ativo ou Reflexivo.

- Tipo de recurso de aprendizagem: Valores como 'texto', 'figura', 'experimento', 'questionário', 'simulação, 'avaliação', etc., indicam o(s) tipo(s) específico(s) do OA e permite identificar se é mais apropriado para estudantes com EAs de três dimensões: Sensitivos/Intuitivos, Visuais/Verbais, Ativos/Reflexivos.

Adicionalmente, os campos Estrutura e Formato da categoria Técnica também são consideradas no mapeamento realizado:

- Estrutura: Este campo sugere a estrutura organizacional subjacente de um OA, e permite verificar se o OA é mais apropriado para um aluno global ou sequencial. 
VI Congresso Brasileiro de Informática na Educação (CBIE 2017)

Anais do XXVIII Simpósio Brasileiro de Informática na Educação (SBIE 2017)

- Formato: Seu vocabulário indica os tipos de dados de um OA ('MIME types') e permite determinar se um OA pode ser recomendado para um estudante Visual, Verbal, Ativo ou Reflexivo.

As recomendações devem sugerir conteúdo com base nas expectativas dos estudantes. Alguns sistemas de recomendação visam auxiliar o usuário na busca e seleção de um conteúdo focado em seu perfil [Cazella et al. 2012], para que o usuário tenha como resultado de busca apenas os materiais mais próximos e relevantes.

Visto que já existe uma grande quantidade de OAs e que novos materiais são criados a cada dia, o elevado volume de conteúdo gerado faz com que técnicas de classificação e agrupamento se tornem um fator de relevância na área de educação, de modo a melhorar o processo de recomendação de conteúdo [Michalski et al. 2013].

\subsection{Clusterização}

A clusterização é o processo de agrupamento de dados que utiliza algoritmos capazes de avaliar a similaridade entre os atributos presentes nos dados. Com isso, pode-se dizer que elementos pertencentes a um determinado cluster (ou grupo) possuem características semelhantes entre si. O agrupamento de dados representa uma abordagem inerente para uma melhor compreensão de todo o conjunto, sendo importante também para organizar conteúdos similares. É importante citar alguns algoritmos que serão utilizados na abordagem proposta:

- K-Means: proposto por [MacQueen et al. 1967] que é um algoritmo que agrupa dados em centroides, criados inicialmente de maneira aleatória, que são reajustados em direção ao centro dos clusters para serem reorganizados a cada iteração.

- Expectation Maximization (EM): [Dempster et al. 1977] calcula probabilidades dos membros dos clusters com base em uma ou mais distribuições de probabilidade. O objetivo do algoritmo é de maximizar a probabilidade global ou a probabilidade dos dados, considerando os grupos finais.

- Self Organizing Map (SOM): a rede SOM, proposta [Kohonen 1998], é uma rede neural de aprendizado competitivo, na qual os neurônios de uma camada competem entre si pelo privilégio de permanecerem ativos, onde um neurônio com maior atividade é o único a participar do processo de aprendizado, criando assim clusters dos dados.

O processo de agrupamento pode resultar em diferentes particionamentos de um conjunto de dados, dependendo do critério específico utilizado como parâmetor. Assim, existe uma necessidade de pré-processamento antes de assumir uma tarefa de agrupamento em um conjunto de dados. Os passos básicos para desenvolver um processo de agrupamento são apresentados nas seguintes etapas: Seleção de Dados, Algoritmo de agrupamento, Validação dos Resultados e Interpretação dos Resultados [Fayyad et al. 1996].

Neste sentido, a estratégia de utilizar técnicas de clusterização para recomendação personalizada de conteúdo para organizar objetos de aprendizagem tem um grande potencial para ser aplicada em ambientes educacionais adaptativos. 
VI Congresso Brasileiro de Informática na Educação (CBIE 2017)

Anais do XXVIII Simpósio Brasileiro de Informática na Educação (SBIE 2017)

\section{Abordagem Proposta}

A abordagem proposta consiste no agrupamento dos OAs com características similares e, a partir desse agrupamento, na posterior recomendação de conteúdo para estudantes, conforme seus EAs correspondentes. O processo de agrupamento se dá a partir da vetorização gerada pelas regras que mapeiam os campos de metadado no formato IEEELOM com os EAs do modelo FSLSM, apresentadas em [Dorça et al. 2016]. Assim, dado um repositório de metadados no formato indicado, as regras são avaliadas e um vetor é gerado para o metadado avaliado. Esses vetores são submetidos ao processo de clusterização que identifica os grupos de OAs similares, conforme mostrado na Figura 1.

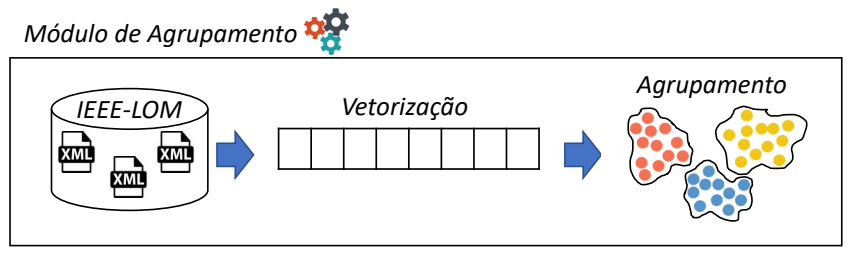

Figure 1. Processo de Agrupamento de OAs.

Para tornar possível o agrupamento, foi criado um método de vetorização com base nos atributos dos OAs que consiste em contabilizar a quantidade de regras satisfeitas para cada EA. Por exemplo, um OA que atende duas regras relacionadas com o EA Sensitivo, uma regra relacionada com o EA Ativo, três regras relacionadas com o EA Reflexivo, uma regra relacionada com o EA Global e duas regras relacionadas com o EA Verbal, resultará no vetor apresentado na Tabela 1. Esse vetor possui oito parâmetros indicando cada um dos EAs do modelo FSLSM: Sensitivo(Sen), Intuitivo(Int), Ativo(Ati), Reflexivo(Ref), Sequencial(Seq), Global(Glo), Visual(Vis) e Verbal(Ver).

Table 1. Vetor de representação de um OA considerando seus EAs.

\begin{tabular}{|l|l|l|l|l|l|l|l|}
\hline \multicolumn{7}{|c|}{ Objeto de Aprendizagem x FSLSM } \\
\hline Sen. & Int. & Ati. & Ref. & Seq. & Glo. & Vis. & Ver. \\
\hline 2 & 0 & 1 & 3 & 0 & 1 & 0 & 2 \\
\hline
\end{tabular}

Para validar os resultados, foi criado um rótulo para cada agrupamento, como exemplo um grupo que está nos EAs de Sensitivo, Ativo, Global e Verbal será representando da seguinte forma SenAtiGloVer. Portanto, nesta abordagem são considerados 16 possíveis clusters executados pelos algoritmos (a partir das 16 possíveis combinações do modelo FSLSM).

Durante o processo de validação dos resultados, foram utilizadas duas ferramentas para auxiliar na execução dos algoritmos, Weka [Hall et al. 2009] e MatLab [Vesanto et al. 1999]. Foram gerados 1600 registros fictícios e consistentes em relação as regras de mapeamento de EAs, aleatórios e sem ordem. Além disso, foram gerados 100 elementos para cada combinação de EAs.

\section{Resultados}

Três algoritmos de clusterização foram experimentados: K-Means, EM e SOM. Todos os três algoritmos apresentaram OAs nos 16 clusters que representam as combinações possíveis de EAs. 
VI Congresso Brasileiro de Informática na Educação (CBIE 2017)

Anais do XXVIII Simpósio Brasileiro de Informática na Educação (SBIE 2017)

Durante a execução da rede Neural SOM, foi utilizado um mapa $4 \times 4$, por serem esperados 16 clusters, considerando, assim, que cada neurônio representasse um cluster. Em relação ao KMeans e EM, foram fixados o número de cluster em 16, visto que é necessário informar o número de grupos esperados nesses algoritmos. Com isso, pode-se dizer que houve coerência nos resultados obtidos inicialmente pois todos os OAs foram inseridos nos grupos que eram esperados.

Após a geração dos clusters, a qualidade de cada um deles foi avaliada utilizando métricas de validação como Pureza(P), que valida se existem diferentes elementos em cada cluster, e F-Measure (F), que avalia a qualidade de cada cluster com base nos cálculos de precision e recall [Xiong et al. 2009]. A Tabela 2 apresenta os resultados obtidos em cada métrica para cada um dos algoritmos testados.

Table 2. Validação dos resultados da clusterização

\begin{tabular}{|c|c|c|c|c|c|c|}
\hline \multicolumn{3}{|r|}{ KMeans } & \multicolumn{2}{|r|}{ EM } & \multicolumn{2}{|r|}{ SOM } \\
\hline $\mathbf{C}$ & Qtd & Classes & Qtd & Classes & Qtd & Classes \\
\hline 1 & 100 & SenRefGloVis & 100 & SenRefGloVis & 111 & IntRefSeqVis, IntRefGloVis \\
\hline 2 & 100 & SenRefSeqVis & 100 & IntRefSeqVer & 89 & IntRefGloVis, IntRefSeqVis \\
\hline 3 & 100 & IntAtiGloVis & 100 & SenAtiGloVis & 60 & IntAtiSeqVis, IntAtiGloVis \\
\hline 4 & 100 & IntAtiGloVer & 100 & IntAtiGloVer & 140 & IntAtiGloVis, IntAtiSeqVis \\
\hline 5 & 100 & IntRefGloVer & 100 & IntAtiSeqVer & 116 & SenRefSeqVis, SenRefGloVis \\
\hline 6 & 34 & SenAtiGloVis & 100 & IntRefSeqVis & 84 & SenRefGloVis, SenRefSeqVis \\
\hline 7 & 100 & SenAtiSeqVer & 86 & SenAtiSeqVer & 90 & SenAtiSeqVis, SenAtiGloVis \\
\hline 8 & 66 & SenAtiGloVis & 96 & IntAtiSeqVis & 110 & SenAtiGloVis, SenAtiSeqVis \\
\hline 9 & 119 & IntRefSeqVis, IntAtiSeqVis & 100 & SenRefSeqVer & 104 & SenRefGloVer, SenRefSeqVer \\
\hline 10 & 100 & SenAtiGloVer & 100 & IntRefGloVis & 96 & SenRefSeqVer, SenRefGloVer \\
\hline 11 & 100 & SenAtiSeqVis & 75 & SenAtiSeqVis, IntAtiSeqVis & 74 & SenAtiSeqVer, SenAtiGloVer \\
\hline 12 & 100 & IntRefGloVis & 100 & IntRefGloVer & 126 & SenAtiGloVer, SenAtiSeqVer \\
\hline 13 & 135 & IntAtiSeqVis, IntAtiSeqVer & 100 & IntAtiGloVis & 123 & IntRefSeqVer, IntRefGloVer \\
\hline 14 & 100 & SenRefGloVer & 43 & SenAtiSeqVis, SenAtiSeqVer & 77 & IntRefGloVer, IntRefSeqVer \\
\hline 15 & 146 & IntRefSeqVer, IntAtiSeqVer & 200 & SenAtiGloVer, SenRefGloVer & 58 & IntAtiSeqVer, IntAtiGloVer \\
\hline 16 & 100 & SenRefSeqVer & 100 & SenRefSeqVis & 142 & IntAtiGloVer, IntAtiSeqVer \\
\hline $\mathbf{M}$ & P: 0,945 & F: 0,923 & P:0,945 & F: 0,928 & P: 0,562 & $\mathbf{F}: \mathbf{0 , 5 5 3}$ \\
\hline
\end{tabular}

Como pode ser visto na Tabela 2, ao se comparar a pureza e a qualidade de cada cluster em cada um dos algoritmos testados, o K-Means e o EM obtiveram melhores resultados. Em contrapartida, um alto grau de imprecisão nos clusters foi encontrado quando executado o algoritmo SOM. Por isso, o K-Means e o EM apresentam pontos positivos e favoráveis para a utilização do modelo. Em particular, o EM obteve uma pequena vantagem na métrica F com relação ao K-Means $(0,928$ e 0,923, respectivamente).

Visto isso, conclui-se que a abordagem apresentada mostrou-se promissora para uma recomendação de OAs considerando os EAs do modelo FSLSM. Assim, os OAs de um repositório muito grande podem ser agrupados em características similares que mais se assemelham os EAs dos estudantes para tornar os ambientes virtuais de aprendizagem mais inteligentes e personalizados.

\subsection{Recomendação de OAs Reais}

A abordagem preliminar foi aplicada aos OAs reais gerados pela plataforma de aprendizagem ubíqua chamada de Classroom eXperience (CX) [Araújo et al. 2016]. Nessa plataforma, o professor prepara seus slides, grava sua aula presencialmente com o apoio de dispositivos computacionais, como lousa eletrônica, projetores, notebooks e câmeras, e então as mídias capturadas são disponibilizadas aos estudantes matriculados.

A arquitetura do CX possui módulos responsáveis pela geração de OAs já associados com EAs do modelo FSLSM, representação das características dos estudantes 
VI Congresso Brasileiro de Informática na Educação (CBIE 2017)

Anais do XXVIII Simpósio Brasileiro de Informática na Educação (SBIE 2017)

por meio de um modelo de estudante que também contempla seu EA e um módulo responsável pela personalização e recomendação de OAs.

Durante o primeiro semestre de 2017, foram gerados 865 OAs relacionados com a disciplina Interação Humano-Computador (IHC), ministrada em uma universidade federal brasileira para alunos de graduação em Ciência da Computação e Sistemas de Informação. Os OAs referentes a cada uma das aulas da disciplina foram submetidos ao algoritmo EM, uma vez que o mesmo obteve o melhor desempenho nos experimentos com dados sintéticos. Cada aula gerou, na média, três clusters.

O algoritmo também foi executado considerando todos os OAs da disciplina IHC. Nesse cenário, 13 clusters foram gerados no total. A Figura 2 apresenta a quantidade de regras atendidas para cada um dos oito EAs em cada cluster.

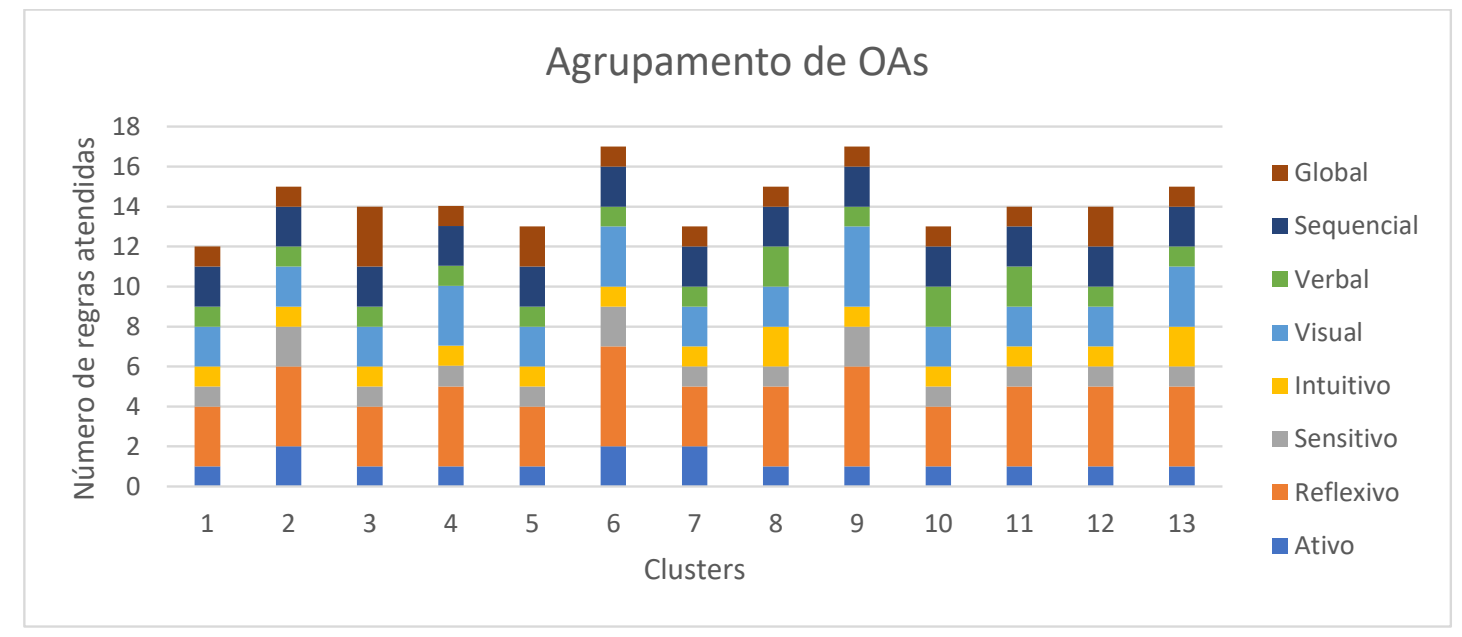

Figure 2. Agrupamento de OAs da disciplina IHC gerados pela plataforma CX.

Com base no agrupamento de OAs realizado, é possível reduzir a quantidade de material a ser selecionado em um processo de recomendação. O ambiente virtual de aprendizagem pode, então, selecionar um dos clusters que mais se enquadra nas características de EA de um estudante em específico para recomendar materiais adicionais de forma individualizada.

Imagine que uma segunda disciplina necessite de materiais adicionais (extra) mas que são relacionados com a disciplina de IHC para sugerir a um determinado estudante com características que acentuam os EAs Reflexivo e Visual. Nesse caso, os OAs presentes no cluster de número 9 poderiam ser selecionados para serem sugeridos. É possível, ainda, executar algum algoritmo de ranqueamento para selecionar apenas os OAs desse cluster que mais fazem sentido naquele momento para o estudante, o que caracterizaria um segundo nível de recomendação.

\section{Trabalhos Relacionados}

No domínio educacional, modelos de recomendação de conteúdo têm sido empregados em diferentes contextos. O modelo proposto em [dos Santos et al. 2015] aplica um algoritmo de filtragem colaborativa em clusters de OAs e de usuários. Foi realizada uma 
VI Congresso Brasileiro de Informática na Educação (CBIE 2017)

Anais do XXVIII Simpósio Brasileiro de Informática na Educação (SBIE 2017)

clusterização com base em conteúdo de duas formas distintas: primeiramente, com os OAs e, em posteriormente, com os usuários, para gerar a recomendação. Entretanto, este modelo não explora a relação entre OAs e EAs para recomendação.

[Cazella et al. 2012] apresenta um sistema de recomendação de OAs baseado na técnica de filtragem colaborativa. O modelo recomenda OAs aos estudantes de forma automática, com base em seus interesses e nas competências que devem ser desenvolvidas seguindo um plano de aula. Sua proposta, no entanto, não utiliza o modelo FSLSM para avaliar o perfil de aprendizagem do estudante.

Casagrande et al. [Casagrande et al. 2015] apresentam uma técnica para avaliar os valores dos elementos de metadados do conteúdo acessado pelos estudandes a fim de estimar as suas preferências e formular a recomendação correspondente. Seu objetivo é determinar as preferências do estudante a partir de dados que descrevem o conteúdo acessado pelo mesmo. O conjunto de elementos de metadados a ser observado é dependente do domínio do repositório, permitindo que metadados descritores de objetos de aprendizagem sejam considerados.

A Tabela 3 apresenta um comparativo da abordagem proposta frente aos principais trabalhos relacionados. O trabalho ora apresentado se destaca por realizar agrupamentos de acordo com os EAs atendidos pelos OAs, considerando as 16 combinações possíveis de EAs dentro do modelo de Felder e Silverman. Tal abordagem propõe uma forma vetorial de representar os OAs considerando os EAs que eles atendem, tornando o processo de recomendação por EAs muito mais eficiente. Ainda, com o resultado desta vetorização, torna-se possível aplicar técnicas de agrupamento que, por serem independentes de um repositório ou AVA específico, contribuem para o processo de recomendação personalizada de conteúdo. A única restrição é que OAs tenham metadados segundo o formato IEEE-LOM.

Table 3. Comparativo da abordagem proposta com trabalhos relacionados.

\begin{tabular}{lccccc}
\hline \multicolumn{1}{c}{ Trabalhos } & Cluster OAs & Cluster Usuários & FC & FSLSM & IEEE LOM \\
\hline Casagrande et al. (2015) & & $\mathrm{x}$ & & & \\
dos Santos et al. 2015 & $\mathrm{x}$ & $\mathrm{x}$ & $\mathrm{x}$ & & \\
Cazella et al. 2012 & & & $\mathrm{x}$ & & \\
Este trabalho & $\mathrm{x}$ & & & $\mathrm{x}$ & $\mathrm{x}$ \\
\hline
\end{tabular}

\section{Conclusão}

$\mathrm{O}$ fato de as pessoas se comportarem e aprenderem em ritmos diferentes requer que as diferenças individuais dos estudantes sejam consideradas no processo de aprendizagem. A utilização de OAs e EAs para recomendação de conteúdo pode facilitar esse processo, apoiando professores e estudantes nas respectivas atividades de produção e consumo de conteúdo.

Este artigo apresentou uma abordagem de recomendação de conteúdo educacional que utiliza técnicas de agrupamento de dados para agrupar OAs em repositórios com base no modelo FSLSM. A abordagem proposta utiliza recursos de clusterização de vetores que representam metadados de OAs no formato IEEE-LOM associados aos EAs dos estudantes. 
VI Congresso Brasileiro de Informática na Educação (CBIE 2017)

Anais do XXVIII Simpósio Brasileiro de Informática na Educação (SBIE 2017)

A partir de dados reais coletados na plataforma Classroom eXperience, foi realizada uma análise comparativa de três diferentes algoritmos de agrupamento, cujos resultados permitiram identificar a melhor técnica (EM) utilizando métricas de validação consistentes. O algoritmo foi então executado em um conjunto de 865 OAs de uma disciplina de graduação, produzindo 13 clusters e permitindo ao AVA recomendar o que melhor se enquadrasse ao perfil do estudante com relação ao seu EA.

Com isso é possível facilitar o processo de recomendação personalizada de conteúdo considerando EAs em ambientes educacionais, trazendo vantagens em termos de eficiência, uma vez que a abordagem possibilita o pré-processamento das características dos EAs atendidos pelos OAs.

\section{Agradecimentos}

Os autores agradecem o apoio recebido do CNPq, da CAPES, da FAPEMIG, da PROPP/UFU, da FACOM/UFU e do PET/MEC/SESu para realização deste trabalho.

\section{References}

Almeida, M. E. B. d. (2003). Educação a distância na internet: abordagens e contribuições dos ambientes digitais de aprendizagem. Educação e Pesquisa, 29:327-340.

Araújo, R. D., Brant-Ribeiro, T., Ferreira, H. N. M., Dorça, F. A., and Cattelan, R. G. (2016). Segmentação Colaborativa de Objetos de Aprendizagem Utilizando Bookmarks em Ambientes Educacionais Ubíquos. In Anais do 27º Simpósio Brasileiro de Informática na Educação, SBIE'16, pages 1205-1214, Uberlândia, MG, BR. SBC.

Audino, D. F. and da Silva Nascimento, R. (2012). Objetos de aprendizagem-diálogos entre conceitos e uma nova proposição aplicada à educação. Revista Contemporânea de Educação, 5(10).

Casagrande, M. F. R., Kozima, G., and Willrich, R. (2015). Técnica de recomendação baseada em metadados para repositórios digitais voltados ao ensino. Revista Brasileira de Informática na Educação, 23(02):70.

Cazella, S. C., Behar, P., Schneider, D., da Silva, K. K., and Freitas, R. (2012). Desenvolvendo um sistema de recomendação de objetos de aprendizagem baseado em competências para a educação: relato de experiências. In Anais do Simpósio Brasileiro de Informática na Educação, volume 23.

Cazella, S. C., Reategui, E., Machado, M., and Barbosa, J. (2009). Recomendação de objetos de aprendizagem empregando filtragem colaborativa e competências. Simpósio Brasileiro de Informática na Educação (SBIE).

Dempster, A. P., Laird, N. M., and Rubin, D. B. (1977). Maximum likelihood from incomplete data via the em algorithm. Journal of the royal statistical society. Series B (methodological), pages 1-38.

Dorça, F. A., Araújo, R. D., de Carvalho, V. C., Resende, D. T., and Cattelan, R. G. (2016). An Automatic and Dynamic Approach for Personalized Recommendation of Learning Objects Considering Students Learning Styles: An Experimental Analysis. Informatics in Education, 15(1):45-62. 
VI Congresso Brasileiro de Informática na Educação (CBIE 2017)

Anais do XXVIII Simpósio Brasileiro de Informática na Educação (SBIE 2017)

dos Santos, H., Cechinel, C., Araújo, R., and Brauner, D. (2015). Recomendação de objetos de aprendizagem utilizando filtragem colaborativa: Uma comparação entre abordagens de pré-processamento por meio de clusterização. In Anais do Simpósio Brasileiro de Informática na Educação, volume 26, page 1127.

Fayyad, U. M., Piatetsky-Shapiro, G., Smyth, P., and Uthurusamy, R. (1996). Advances in knowledge discovery and data mining.

Felder, R. M. and Silverman, L. K. (1988). Learning and teaching styles in engineering education. Engineering education, 78(7):674-681.

Hall, M., Frank, E., Holmes, G., Pfahringer, B., Reutemann, P., and Witten, I. H. (2009). The weka data mining software: an update. ACM SIGKDD explorations newsletter, 11(1):10-18.

Hodgins, W., Duval, E., et al. (2002). Draft standard for learning object metadata (1484.12.1). Learning Technology Standards Committee of the IEEE.

Kohonen, Teuvo, P. (1998). Self-organizing maps of symbol strings. Neurocomputing, 21(1):19-30.

MacQueen, J. et al. (1967). Some methods for classification and analysis of multivariate observations. In Proceedings of the fifth Berkeley symposium on mathematical statistics and probability, volume 1, pages 281-297. Oakland, CA, USA.

Michalski, R. S., Carbonell, J. G., and Mitchell, T. M. (2013). Machine learning: An artificial intelligence approach. Springer Science \& Business Media.

Riding, R. and Rayner, S. (2013). Cognitive styles and learning strategies: Understanding style differences in learning and behavior. Routledge.

Torres, T. Z. and do Amaral, S. F. (2011). Aprendizagem colaborativa e web 2.0: proposta de modelo de organização de conteúdos interativos. Educação Temática Digital, 12:49.

Vesanto, J., Himberg, J., Alhoniemi, E., Parhankangas, J., et al. (1999). Self-organizing map in matlab: the som toolbox. In Proceedings of the Matlab DSP conference, volume 99, pages 16-17.

Weiser, M. (1999). The computer for the 21st century. SIGMOBILE Mob. Comput. Commun. Rev., 3(3):3-11.

Xiong, H., Wu, J., and Chen, J. (2009). K-means clustering versus validation measures: a data-distribution perspective. Systems, Man, and Cybernetics, Part B: Cybernetics, IEEE Transactions on, 39(2):318-331.

Zaina, L., Bressan, G., Cardieri, M., and Júnior, J. F. R. (2012). e-lors: Uma abordagem para recomendacao de objetos de aprendizagem. Revista Brasileira de Informática na Educação, 20(1):04. 